\title{
The Co-Retweeted Network and its Applications for Measuring the Perceived Political Polarization
}

\author{
Samantha Finn ${ }^{1}$, Eni Mustafaraj ${ }^{1}$ and Panagiotis T. Metaxas ${ }^{1}$ \\ ${ }^{1}$ Department of Computer Science, Wellesley College, Wellesley, MA02481, USA \\ \{sfinn,emustafa, pmetaxas\}@wellesley.edu
}

\begin{abstract}
Keywords: $\quad$ Social Media Analytics, Social Networks, Twitter, Big Data, Social and Legal Issues, Human Computation.
Abstract: This paper introduces a novel network, the co-retweeted network, that is constructed as the undirected weighted graph that connects highly visible accounts who have been retweeted by members of the audience during some real-time event. Like bibliographics co-citation used to indicate that two papers treat a related subject matter, co-retweeting is used to indicate that two accounts present similar opinions in an online discussion. Thus, the co-retweeted network can be seen as a form of consulting the opinion of the crowd that is following the discussion about the similarity (or difference) of positions expressed by the highly visible accounts. When applied on political conversations related to some event, the co-retweeted network enables the measurement of the polarity of political orientation of major players (including news organizations) based on the views of the audience. It can also measure the degree of polarization of the event itself.
\end{abstract}

\section{INTRODUCTION}

Presidential debates in the United States are very important political events. Their television audience consistently ranks among the largest of the year (second only to the famous "Super Bowl", the championship game of US Football). However, these spectators are no longer passive: they increasingly use the web as a platform for further engagement. As studies from Pew Research have shown, 1 in 10 spectators in presidential debates is a "dual-screener" 1 . Very often the second screen is Twitter, where running commentary of live-televised events is at its liveliest.

But studying such lively online discussions has a significant obstacle: data size. During the 2012 US presidential race President Barack Obama and challenger Mitt Romney held three debates on October $3 \mathrm{rd}, 16 \mathrm{th}$ and $22 \mathrm{nd}$. These debates generated respectively 10.3 million $^{2}, 7.2$ million $^{3}$, and 6.5 million $^{4}$ tweets in a time span of approximately $90 \mathrm{~min}$. each.

Currently, only Twitter (the company) is able to make sense of such big data by creating simple histogram-like data aggregations that spike during the debates (charts can be found in footnotes 2, 3, 4). By

\footnotetext{
${ }^{1}$ Social media a hotbed of political debate, engagement - for the good? http://to.pbs.org/1bCvUoJ

${ }^{2}$ Dispatch from the Denver debate, http://bit.ly/1k15reF

${ }^{3}$ Twitter at the Town Hall Debate, http://bit.ly/1kWkBj6

${ }^{4}$ The Final Presidential Debate, http://bit.ly/19Av3VM
}

establishing a correspondence between what was being said during the debate and the number of tweets per minute mentioning those words (and other debaterelated hashtags), Twitter can quantify how moments during the live event affected the tweeting public.

While this is a good way to summarize the major issues tweeted during an event, one might be interested not only in what is being tweeted, but also who is tweeting it and why. In addition, there are other questions that often occupy the public discourse during political discussions: How polarized were the political postings online? Did the major news organizations take sides in favor of one or the other candidate? And did the supporters of one discussant were more vocal than the supporters of the other?

News organizations answer these questions by using human experts who try to make sense of the data, preferably while the event is developing. However, no matter how well trained, human experts will hardly be able to keep track of the avalanche of data coming at a speed of 100,000 tweets/minute. Our approach instead is to crowd source the solution by using "human computation" (von Ahn et al., 2006) in the form of analyzing the independent decisions made by large groups of users in parallel. In Twitter, this takes into account retweets ${ }^{5}$ and favorites.

\footnotetext{
${ }^{5}$ In this paper, when we refer to "retweets" we mean verbatim retweets created by the clicking of the retweet button, not the manually created modified tweets.
} 
In an effort to take advantage of this human computation, we propose the construction of a novel network, the co-retweeted network of Twitter accounts. Our insight is that the co-retweeted network displays some dynamics of Twitter conversations during a certain event as revealed by the repeated and independent behavior of the millions of users following the event. We define as co-retweeting the act of a single user retweeting two -or more- different accounts. These acts are used to create undirected weighted edges between the co-retweeted accounts in the network. The more users retweet these two accounts, the more weight the edge gains. And there can be a lot of retweets to include in the network construction. In fact, the data we collected during the presidential debates show that more than $50 \%$ of the tweets sent are retweets. However, some tweets will have greater visibility, receiving in the order of several thousand retweets, while the vast majority of them will only receive one or two retweets. We use this information to create a co-retweeted network that captures how the wider public views the major participants in the discussion. An example visualizing the second presidential debate using the co-retweeted network is shown in Figure 1. To the best of our knowledge, this has not been previously studied in the Twitter-related research.

We claim that the co-retweeted network is able to measure the perceived political orientation of the major players by recording the retweets of the audience on Twitter. Co-retweeting can be compared to the concept of co-citation (Small, 1973). The latter is a well known bibliometrics measurement used by librarians to determine whether two published works may be treating similar subjects or not. When there are a lot of works co-citing two papers, A and B, this is considered evidence that A and B treat related subjects. In this respect, it reflects the opinion of many authors and thus represents a better indicator of subject similarity. The more co-citations two documents receive, the more likely they are semantically related. (In fact, reviewers of papers enforce that by demanding the inclusion of missing co-citations.)

We use co-citation in the same spirit but, in this paper, in a more restricted domain: During a political event, when lots of Twitter users retweet two accounts, $U_{A}$ and $U_{B}$, we consider it as evidence that $U_{A}$ and $U_{B}$ are taking politically similar positions. The more co-retweeting the tweets of $U_{A}$ and $U_{B}$ receive, the more likely they are politically related. This fact can lead to some concrete measurement on how polarized two accounts are and, when considering the overall political interaction, how polarized the event is. Effectively, we are able to compute the answers to the three questions we posed above.

The remaining of this paper is organized as follows. After describing prior work related to the issues treated in this paper, we define the co-retweeted network and describe its construction. Then we present the applications we consider in this paper, measuring the degree of political polarity during an online event, and measuring the polarity of the major players participating in the event. We end with our conclusions.

\subsection{Prior Work}

Co-citation was first introduced by Henry Small (Small, 1973) and is considered as a basic concept in Bibliometrics. The histogram-like data aggregations mentioned in the introduction was first applied by researchers during a debate for the 2008 US Elections (Diakopoulos and Shamma, 2010).

We introduced the concept of co-retweeting in (Finn and Mustafaraj, 2013), with a possible application for recommending relevant tweets during an event. The domain of recommendation and specifically the algorithms for item-based collaborative filtering are at the foundation of our method for building the co-retweeted network, which follows the principles of building the item-item similarity matrix with co-rated items (Sarwar et al., 2001). In the current paper, we expand the definition of the co-retweeted network and introduce new applications for it.

The first application, measuring opinion polarization for an issue or topic, is a research question that is usually treated in the political sciences literature. A review of the literature on this question (Fiorina and Abrams, 2008) concluded that the "American public as a whole is no more polarized today that it was a generation ago". While these studies focus on the public as a whole, we are posing this question in the context of the social media participants, which might not be representative of the entire public but still can command certain influence over the public discourse and its coverage in the traditional media. Furthermore, since Twitter has a global userbase, we can measure polarity in political discourse in other countries (e.g. Germany) as well.

The second application of the co-retweeted network is about calculating an account's polarity and using it to measure the media bias (as perceived by the Twitter audience). Researchers have always been concerned with media bias for a long time (for an overview refer to (Prior, 2013)) and different research fields use various methodologies to measure it. A somewhat related approach that makes use of Twitter, but doesn't calculate media bias (it calculates the political preferences of a (media) organization's Twit- 


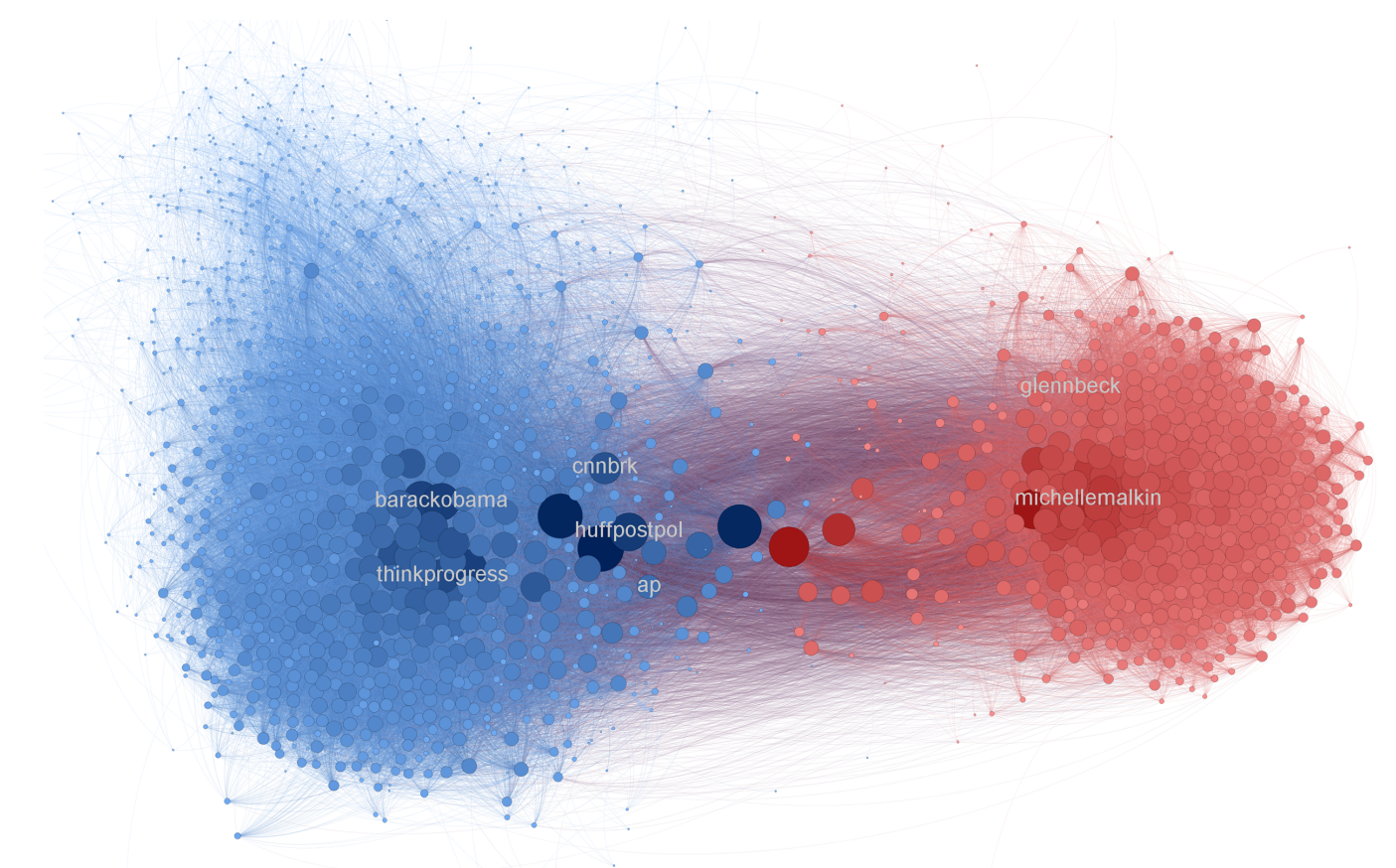

Figure 1: The network visualization for the 1,500 most co-retweeted accounts during the second debate. It turns out that, in this data set, the nodes colored blue by the community detection algorithm belong to accounts with a liberal political orientation and the nodes colored red to accounts with conservative political orientation. The labels indicate the location of some of the more visible accounts belonging to news organizations and political entities.

ter followers instead) can be found in (Golbeck and Hansen, 2014). Their method relies on the static relation of "followship". Our method instead is dynamic in the sense that every co-retweeted network is eventspecific and may display different bias. Such specificity cannot be captured through static relations.

Additionally, we use the account polarity method to infer the polarity of all (engaged in retweeting) accounts in the audience to answer the question of whose supporters were more active and vocal during an event. This problem has certain similarities to the task of classifying Twitter users based on their political orientation as described in (Conover et al., 2011) and (Cohen and Ruths, 2013), however, our approach differs significantly from theirs. The cited research uses machine learning to classify users who have been represented by a set of features (mostly hashtags and other metadata from their tweets). We don't use the text of the tweets in our approach. Instead, the polarity (or orientation) is calculated based on the characteristics of the co-retweeted network, as well as the retweeting behavior of the users. A limitation of our method is that it cannot be used for users who didn't retweet. However, previous research has already established that the most vocal supporters engage heavily in retweeting (Mustafaraj et al., 2011; Jungherr et al., 2012).

\section{THE CO-RETWEETED NETWORK}

\subsection{Data Collection}

Collecting data from Twitter is a relatively easy task, due to the different APIs offered by Twitter. However, being sure that one has collected all the relevant or existing data is difficult to evaluate. The only way to be sure is to have access to the Twitter Firehose API, which is usually unreasonably expensive for academic researchers. Currently, researchers use the public APIs (Streaming, Search, REST) by adopting one (or a combination) of the following three methods (Gerlitz and Rieder, 2013):

1) a selection of topic-relevant hashtags and keywords which is fed to the Streaming or Search API,

2) a network of users and their followers (snowball sampling) for which the REST API collects all tweets, 3) metadata features (location, language, etc.) which are also fed to the Streaming or Search API.

For our study, we use the first method: a selection of topic-relevant hashtags and keywords. Two are the major problems with this method: a) the set of preselected keywords and hashtags might not capture the entirety of the discussions about a topic, and b) once 

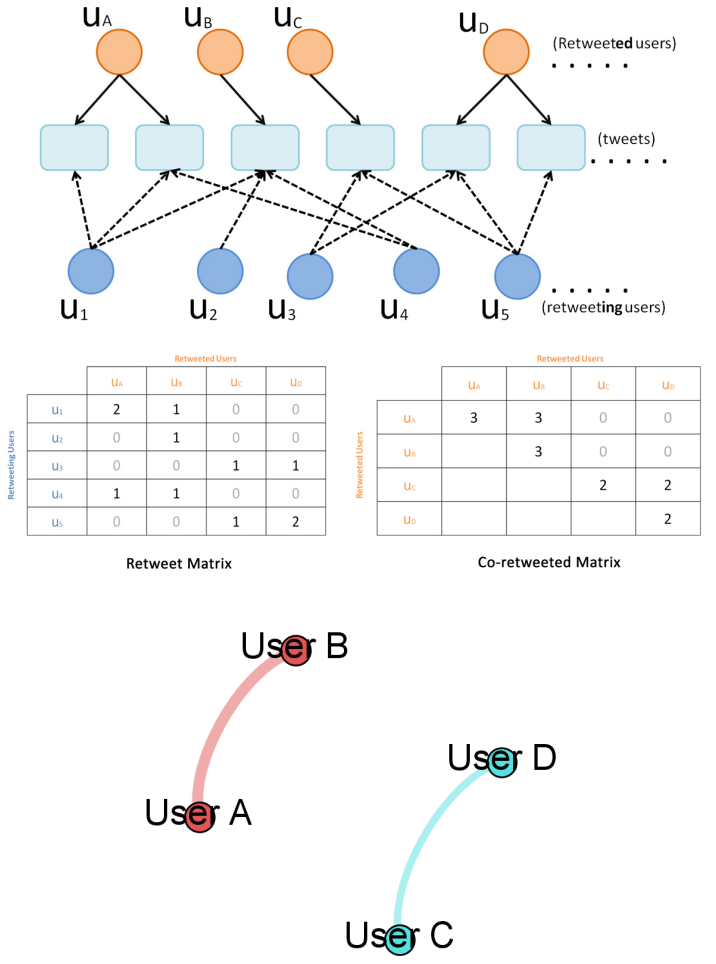

Figure 2: (Top) The process of generating the co-retweeted matrix. The graphic at the top displays the activity of users $U_{A}, U_{B}, \ldots$ whose tweets (blue rectangles) are being retweeted by other users $U_{1}, U_{2}, \ldots$. On the middle left is the corresponding retweet matrix and on the middle right the symmetric co-retweeted matrix. The main diagonal of the co-retweeted matrix shows the number of users retweeting a certain account, and the upper diagonal cells contain the number of times two accounts were co-retweeted. (Bottom) The co-retweeted network produced by the matrix above.

a topic of conversation becomes popular, Twitter applies rate limitations. All data collections have to deal with the first problem, while the second one applies only to conversations that become dominant (such as the US presidential debates discussed in this paper).

Our strategy for dealing with the first problem was the following: during the first presidential debate (Oct. 3, 2012) we used the keywords "obama" and "romney" to collect tweets mentioning the two contenders. The text of tweets was then processed to find the set of most common keywords and hashtags, which then was used for the two successive debates. Additionally, before every new debate we updated the list with hashtags being promoted before the TV broadcast such as \#cnndebate, \#lynndebate, etc. This strategy still doesn't ensure that we will be able to capture the whole conversation, but, for the purposes of our method, we don't need all tweets, we need the tweets that receive the most retweets. Previ- ous research (Suh et al., 2010) has established that "a tweet with hashtags is more likely to get retweeted". By using the most popular hashtags around which the large community has coalesced, we are making the assumption that users who want to be retweeted will be using these agreed upon hashtags. Furthermore, our previous experience with collecting election data from Twitter (Mustafaraj et al., 2011) has shown that Twitter users who play the curator role, will add the appropriate hashtags before retweeting a message relevant to a conversation.

The problem of Twitter rate limits appears whenever the conversation about a topic or event exceeds the $1 \%$ of the whole Twitter volume. The presidential debates, which often averaged at 100,000 tweets/minute ${ }^{6}$ were some of the most-tweeted events in the Twitter history (the first debate was the mosttweeted event ever). Thus, we know that we were able to capture only a small fraction of the sent tweets (in fact, Twitter includes a message in the Streaming API to indicate how many tweets were missed since the latest received tweet, e.g., "limit": "track" : 1234 , and we can calculate how many tweets we missed). Since we are mostly interested in popular retweets, one way to mitigate the rate limitation during the live TV coverage of the debates was to continue the data collection several hours beyond the event completion. This makes sure that we get retweets of content that users who didn't follow the live debate found interesting afterwards. We then tested that the 1,500 tweets with most retweets in our sample are a good representation of most retweeted content by the following procedure: in a later date, we recollected the top 3,000 tweets from our dataset, checked their retweet count, and ranked them based on this number. By comparing these two rankings, we found that our sample has $83 \%$ of the top 500 most retweeted tweets, $74 \%$ of top 1000 , and $71 \%$ of top 1500 , offering a good coverage, despite the limited sampling. Finally, recent research on comparing the Twitter Streaming API to the Firehose (Morstatter et al., 2013) has indicated that for network-level measures (such as centrality, etc.) there is correlation between the results of the two datasets. So, while our data collection method might not be perfect, we have taken into account all available measures to overcome the known problems.

\subsection{Co-retweeted network construction}

From the tweets collected as described above, we focus only on tweets with retweet information using the

\footnotetext{
${ }^{6}$ Tracking the \#Debates: From Big Bird to Bayonets http://bit.ly/1dhigZ9
} 
retweeted_status field included in the JSON representation of a tweet. Such information allows us to create pairs of the original message sender and the retweeter. This information is necessary for creating the co-retweeted network and this process is summarized in Figure 2. We create a matrix of retweet relationships, where each row represents a user, and the columns represent users who have been retweeted. From this matrix we obtain the co-retweeted matrix, containing only the users who were retweeted (the columns in the retweet matrix). Note that the coretweeted matrix is symmetric, corresponding to a weighted undirected network, and we show only its upper diagonal.

Each co-retweeted matrix entry represents how many times the two users have both been retweeted by other users. For example, referring to Figure 2, in the retweet matrix, Users $U_{A}$ and $U_{B}$ have both been retweeted by a third user, User $U_{1}$. The entry for $U_{A}$ and $U_{B}$ in the co-retweeted matrix is incremented by 1 . The same is true for $U_{4}$ who has also retweeted both $U_{A}$ and $U_{B}$ ). Using this matrix we create the coretweeted network of the retweeted accounts, where each user in the rows and columns of the matrix becomes a node, with an edge between them weighted by the number of users who have retweeted both of them. Accounts $U_{i}$, for numeric $i$, who have not been retweeted are ignored in the co-retweeted matrix.

\subsection{Visualization of the Oct 16 Debate}

Figure 1 shows the visualization ${ }^{7}$ of the top 1,500 most co-retweeted accounts built using about 1.3 million tweets that we collected as described. This visualization was created using $\mathrm{Gephi}^{8}$ that was chosen for convenience as it includes several useful implementations of community detection, force-directed layout and influence calculations. Other implementations are possible, of course, but using a comprehensive set of implementations was beyond the scope of this paper. After all, using comparable implementations the essence of the co-retweeted network should not change. The co-retweeted network in Figure 1 is layed out using Gephi's ForceAtlas2 implementation (Gephi, 2010) where nodes are attracted to each other based on the cardinality of their connections (Fruchterman and Reingold, 1991).

We notice two very distinct groups, with only a

\footnotetext{
${ }^{7}$ For an interactive version of the Oct. 16 debate, visit http://bit.ly/1d3MRJM and for the Oct 22 debate visit http://bit.ly/1fWajvH. Due to the size of the network and depending on the available bandwidth, loading the page may take a minute.

${ }^{8}$ The Gephi Visualization Tool, http://gephi.org
}

few nodes bridging the gap between them. The colors used in the visualization make these two groups more apparent and are based on the Louvain community finding algorithm (Blondel et al., 2008) which computes groups of nodes containing more edges within the group than outside the group. The size and darkness of each node is based on influence in the network indicated by their eigenvalue centrality value. By comparison to Figure 1, Figure 3 shows the retweet network of the top 1,500 accounts which is highly disconnected. The giant component contains only 550 connected nodes; the remaining nodes are not connected to it and are shown on the periphery. This is not surprising, since the most retweeted nodes do not retweet others, making the retweet network of the top accounts not very useful. Details about the datasets are listed in Table 1.

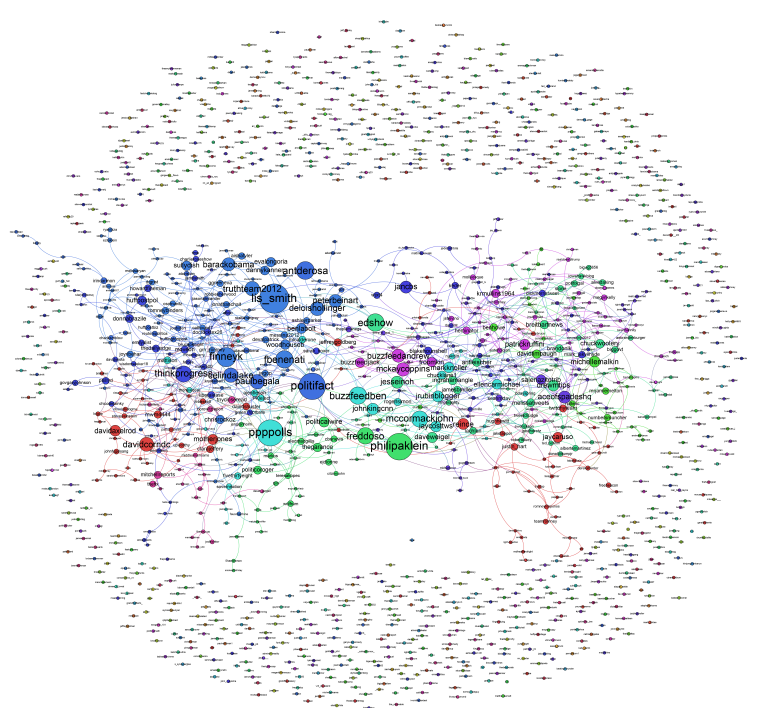

Figure 3: The retweet network of the 1,500 most retweeted users during the debates is not very useful in making sense of the online activity. The central component contains the 550 connected nodes and 6 communities; the remaining components are shown on the periphery. (The full retweet network -not shown here- is also not as useful.)

\section{MEASURING THE PERCEIVED POLITICAL POLARIZATION}

Next we describe a couple of applications for the co-retweeted network. First we describe the insight gained by observing the co-retweeted networks of the two presidential debates. Then we define the measurement "degree of the network polarity" and a method to compute the individual polarity of any member participating in a polarized network. 


\begin{tabular}{|l|c|c|c||c|c|c|c|c|}
\hline & Users & Tweets & Retweets & Nodes & Edges & Modularity & Contrib. Users & Contrib. RTs \\
\hline Oct. 16 & 615,376 & 820,746 & 423,802 & 1,498 & 92,196 & 0.440 & 24,719 & 79,985 \\
Oct. 22 & 646,559 & $1,074,588$ & 582,526 & 1,496 & 126,252 & 0.434 & 45,493 & 179,978 \\
\hline
\end{tabular}

Table 1: Summary of the two debate datasets, which were used to create the co-retweeted networks. Users contributing refers to the number of users who retweeted at least two of the top 1,500 users (in this way they contribute at least one edge to the network). Retweets contributing refers to how many times the top 1,500 users were retweeted by the contributing users.

The visualizations for both presidential debates display two highly separated communities that break down over political lines, with popular news media accounts bridging the gap between them. The distance between the two groups, determined by the force-directed algorithm, is a result of their polarization. The accounts in the blue group on the left of the network are largely liberal leaning politicians and popular bloggers (e.g. @barackobama, @ thinkprogress), while to the right the red consists of conservative accounts (e.g. @glennbeck, @michellemalkin). Between these two groups are media accounts, which are divided between red and blue. Some of these accounts are claiming to be politically unbiased. However, many mainstream news accounts (@ cnnbrk, @ huffpostpol, @ ap) in our event network are classified closer to the blue community.

Previous research has demonstrated how political social media is polarized in the two political orientations (Adamic and Glance, 2005). However, here the situation is different. The links are not created as a result of the actors actively connecting to each-other. In fact, although these are popular accounts, some of whom received thousands of retweets during the debates, they are not active in retweeting each other. In fact, the corresponding retweet graph (Fig. 3) is disconnected. Its giant component shows 6 communities which are not obviously distinguishable, and only the largest two show obvious political orientation. The co-retweeted network bypasses the sparsity of the retweet network by utilizing the hundreds of thousands of retweets made by normal users in order to form connections between the most popular accounts, and by doing this reveals the perceived orientation of the popular accounts. We make use of the assumption (Metaxas and Mustafaraj, 2010; Conover et al., 2011; Wellesley Trails Group, 2014) that the majority of users are not likely to retweet something that disagrees with their political views. Therefore, most of the accounts a single user has retweeted will share a common political bias. We derive the perceived political orientation of an account by the bias of the audience. The co-retweeted network visualizes the political beliefs of the public at large. For example, many news media accounts have been shown to have biased audiences (Golbeck and Hansen, 2011). Fox News and The Drudge Report, which are in the conservative group on our network, have a conservative audience, while National Public Radio and The New York Times have more liberal audiences and appear slightly to the left in our network. Our findings confirm the findings of (Golbeck and Hansen, 2011), however, our method is more flexible since it computes bias on specific events, not in general.

\subsection{Network Polarity}

A polarized network is composed of two or more groups of densely connected nodes that are linked through relatively few inter-group edges. We define as a network's polarity degree, $P D$, the ratio of sum of edges contained within each group over the total number of edges present in the network. Let $E_{i}$ be the number of edges connecting nodes within group $i$ and $E_{i j}$ be the number of edges connecting nodes between groups $i$ and $j$. Then, in a network containing $k$ groups, its polarity degree is:

$$
P D=\frac{\sum_{1 \leq i \leq k} E_{i}}{E}
$$

Since $\sum_{1 \leq i \leq k} E_{i}=E-\sum_{1 \leq i, j \leq k} E_{i j}$, we have

$$
P D=1-\frac{\sum_{1 \leq i, j \leq k} E_{i j}}{E}
$$

Note that $P D$ expresses polarization in a natural way: When the network is composed of few disconnected components (groups of nodes isolated from each other), $P D=1$. On the other hand, when the network is complete, with each node representing a separate highly connected group, $P D=0 .{ }^{9}$

It is well documented that political life in America has become increasingly polarized (Pew Research Center for the People and the Press, 2012). Our analysis and visualization in Fig. 1 shows that this is indeed observed during the electoral debates, and computes its degree of polarization at 0.91 . However, depending on the issue discussed, the polarization degree can rise more than 0.98 , as it happens, for example in the online discussion with the hashtag \#DoYourJobGOP

\footnotetext{
${ }^{9}$ We should note here a limitation of our definition of PD: Intuitively, it is easily justifiable for small $k$ 's, as is the case of parties represented in an election. If $k$ is large, as in the case of family clans, the density of the overall graph should be taken into account.
} 
(See Fig. 4). On the other hand, in Germany, a country where the online discourse was far less polarized than in the US and where coalitions between parties are frequent and expected, the co-retweeted network shows no comparable polarized divisions (Fig. 5). For the recent 2013 German elections co-retweeted network, the polarization degree was 0.48 .

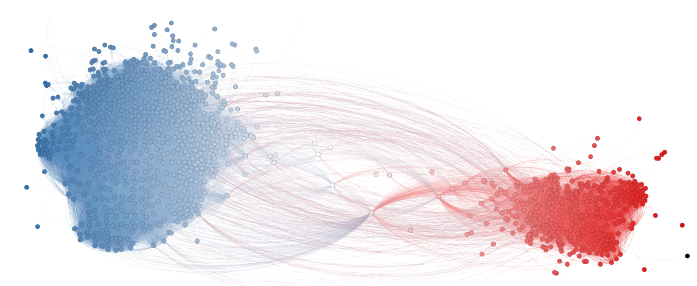

Figure 4: In a highly polarized online discussion in the US, marked by the hashtag DoYourJobGOP (indicating that the Republican party is not performing its duties in the Congress), the degree of polarization was close to $99 \%$. A reason for this higher polarization is, no doubt, the fact that the hashtag was designed to divide as often happens in the so-called hashtag-wars.

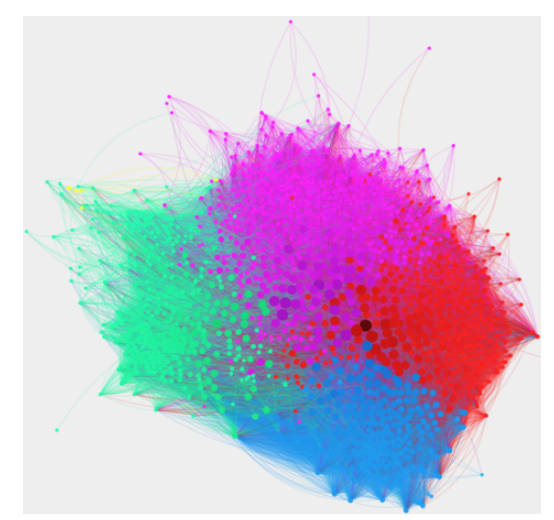

Figure 5: The co-retweeted network for the recent German elections shows far less polarization than during the recent US elections. The network's degree of polarization was about $48 \%$.

\subsection{Computing Account Polarity}

Another related application of the co-retweeted network is to measure the polarity of political orientation for popular accounts according to how the audience sees their messages, not according to their own claims. The co-retweeted network is divided in two groups that exhibit liberal and conservative bias. Less polarized media actors, such as mainstream news media accounts, are retweeted by users of differing political bias and have co-retweeted links between accounts from both the liberal and conservative clusters in the network. In contrast, highly polarized accounts, such as political candidates, are less likely to be retweeted by users with opposing views and the majority of their links will be within their own cluster. This results in certain accounts being drawn into the center of the co-retweeted network, while others end up on the periphery of either the liberal or conservative groups. We utilize this observation to calculate the polarity degree of individual actors in the co-retweeted network.

Let us take the Oct. 16 co-retweeted network as an example for our description below. We define an account's polarity value for a particular network to be the normalized Euclidean distance of the account's node to a center of gravity of the network that typically falls between the two modules. (We normalize on a scale of -1 to 1 , where the center 0 divides conservatives and liberals.) As a result, each account is assigned a polarity value based on their position in the network, as shown in Figure 6.

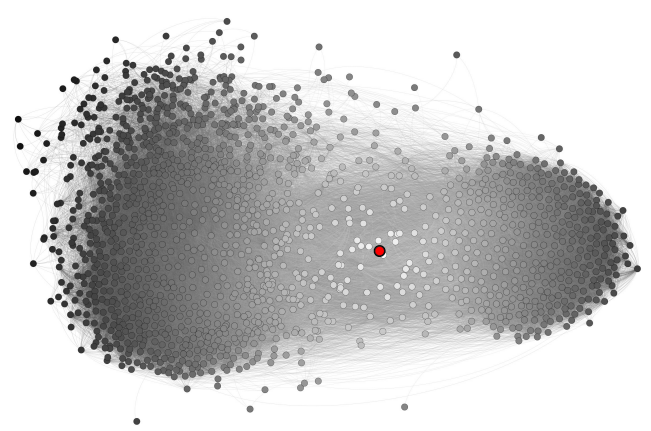

Figure 6: The co-retweeted network for October 16th, where nodes are darkened based on their polarity (darker nodes are more polarized). The large red dot represents the center.

From these polarity values, we can determine the polarity of individual accounts. For every user not included in this network who has retweeted at least two accounts in the co-retweeted network, we calculate their polarity as the weighted average of the polarities of the users they retweeted. A distribution of the 90,000 users for whom we calculated a polarity value shows that the Oct. 16 debate had a largely liberal leaning general audience (see Figure 7 (left)). However, if we look at the more active users that are likely more closely linked to the party's line than the rest, we see a more balanced audience: those who retweeted at least 10 accounts seem to be evenly distributed between the two parties (see Figure 7 (right)). We conclude that, even though both parties were actively trying to inject information in Twitter, the audience retweeted mostly the liberal messages. One could see this as an indication on which speaker in the debates was considered more popular by the online audience. 
To compare the accuracy of the calculated polarities between the retweeted and co-retweeted networks, we performed the same computation in the retweet network and we looked for some ground truth. We manually studied users who had words in their Twitter account description indicative of their political bias (ie, explicitly mentioning that they were democrat, republican, liberal, conservative). We randomly selected 77 such users with opposing polarities, and found that in 75 cases their polarity value accurately described them in the co-retweeted network. We take this as an indication that, even though both retweet and co-retweeted networks may be useful in determining political orientation, the co-retweeted network seems to be a bit more accurate in the determination.

The computation of the center was performed in an automatic calculation as follows. We first used the K-means algorithm to detect two clusters, and counted the cardinalities $V_{l}$ and $V_{r}$ of the left and right cluster. We also determined the center of gravity of each cluster $c_{l}$ and $c_{r}$ approximating them as circles. Finally, we computed the center of the whole system $C$ as the symmetric center of gravity of the whole network, by placing it in distance relative to the ratio of the square root of the cardinalities. The $x$-coordinate of $C$ is computed as:

$$
x_{C}=x_{l}+\frac{\sqrt{V_{r}}}{\sqrt{V_{r}}+\sqrt{V_{l}}} \cdot\left(x_{r}-x_{l}\right)
$$

where $V_{r}$ and $V_{l}$ are the cardinalities ("volumes") of the right and left clusters. The $y$-coordinate is computed similarly.

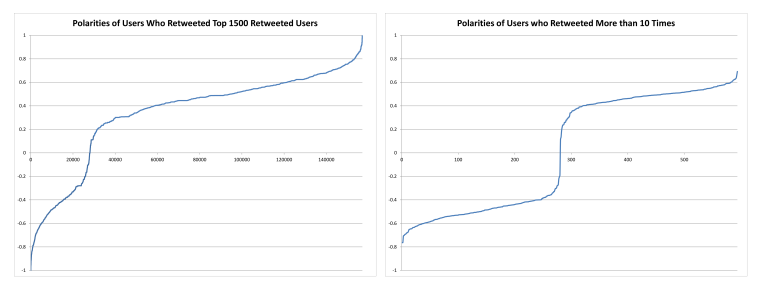

Figure 7: The distribution of polarity among (left) users who retweeted accounts in the co-retweeted network, and (right) those who retweeted accounts at least 10 times. It appears that, in this discussion while the audience was retweeting much more heavily the liberal tweets, the most active members of the audience were equally divided.

\section{CONCLUSIONS}

In this paper we introduced a novel network, the coretweeted network. This is a meta-network that captures aggregated user behavior observed in Twitter during major real-time events. The core power of this network is that it can built on the collective intelligence of hundreds of thousands of humans who, with their retweeting decisions evaluate the major accounts tweeting these real-time events. We also presented two applications: the computation of the polarity of the event itself (as is represented by the network); and the computation of the polarity of the major accounts participating in the discussion (as perceived by the participating audience). Both of them are novel contributions in analyzing online political communication.

In the process we were able to answer the three questions that often are asked during political events: The computed polarity degree is able to answer the question of just how polarized the political postings during an online event were. In particular we found that the 2012 US presidential debates were highly polarized, much more than the discussion around the 2013 German elections, but not as much as the discussions around some online hashtag-wars designed to irritate the opponents and stir controversy.

The computation of a highly visible account's polarity, on the other hand, is able to answer the question on whether major news organizations take sides in favor of one or the other candidate during the debates. This computation is done per event, and not in general as other researchers have done in the past. This is important because news organizations do not always have a definite liberal or conservative approach for every issue. Their stance usually depends on the issue.

And finally we were able to answer the question on whether the supporters of one debater were more vocal than the supporters of another, by looking at the distribution of users' polarities retweeting liberal or conservative messages. We found that for the 2012 debates the answer is more nuanced than a simple yes or no: The distributions reveal that, while the core supporters may have worked equally hard to promote the message of their candidate, the audience was far be less divided in their support.

The concept of co-retweeting, inspired by bibliometrics co-citation and introduced in this paper, can incorporate important aspects of human computation power into the study and analysis of large online social media data. There are other applications one may find, such as its use in a recommendation system, however, describing it is outside the focus of this paper. 


\section{ACKNOWLEDGMENTS}

The authors would like to thank Andreas Jungherr and Pascal Jürgens for providing some of the data from the German elections and for insightful comments on an earlier version of the paper. This research was partially supported by NSF grant CNS-1117693.

\section{REFERENCES}

Adamic, L. A. and Glance, N. (2005). The political blogosphere and the 2004 US election: divided they blog. In Proc. of the 3rd Intl workshop on Link discovery, pages 36-43.

Blondel, V. D., Guillaume, J.-L., Lambiotte, R., and Lefebvre, E. (2008). Fast unfolding of communities in large networks. Journal of Statistical Mechanics: Theory and Experiment, 10.

Cohen, R. and Ruths, D. (2013). Classifying political orientation on twitter: Its not easy! In Proc. of 7th ICWSM. AAAI.

Conover, M. D., Gonçalves, B., Ratkiewicz, J., Flammini, A., and Menczer, F. (2011). Predicting the political alignment of twitter users. In $20113 \mathrm{rd}$ IEEE SocialCom, pages 192-199.

Diakopoulos, N. and Shamma, D. A. (2010). Characterizing debate performance via aggregated twitter sentiment. In CHI, pages 1195-1198.

Finn, S. and Mustafaraj, E. (2013). Visualizing co-retweeting behavior for recommending relevant real-time content. In Proc. of the 4th Intl Workshop on Modeling Social Media, MSM'13, pages 4:1-4:2. ACM.

Fiorina, M. P. and Abrams, S. J. (2008). Political polarization in the american public. Annual Review of Political Science, 11:563-588.

Fruchterman, T. M. J. and Reingold, E. M. (1991). Graph drawing by force-directed placement. Software: Practice and Experience, 21:11291164.

Gephi (2010). ForceAtlas2, the new version of our home-brew layout. http://bit.ly/1deeWht.

Gerlitz, C. and Rieder, B. (2013). Mining one percent of twitter: Collections, baselines, sampling. $M / C$ Journal, 16(2).

Golbeck, J. and Hansen, D. (2011). Computing political preference among twitter followers. In Proc. of the SIGCHI Conf. on Human Factors in Computing Systems, CHI '11, pages 1105-1108, New York, NY, USA. ACM.
Golbeck, J. and Hansen, D. (2014). A method for computing political preference among twitter followers. Social Networks, 36:177-184.

Jungherr, A., Jürgens, P., and Schoen, H. (2012). Why the pirate party won the german election of 2009 or the trouble with predictions: A response to Tumasjan, A., Sprenger, T. O., Sander, P. G., \& Welpe, I. M. "Predicting elections with Twitter: What 140 characters reveal about political sentiment". Social Science Computer Review, 30(2):229-234.

Metaxas, P. T. and Mustafaraj, E. (2010). From obscurity to prominence in minutes: Political speech and real-time search. In Proc. of the WebSci10: Extending the Frontiers of Society OnLine, April 26-27th, 2010, WebScience' 10.

Morstatter, F., Pfeffer, J., Liu, H., and Carley, K. M. (2013). Is the sample good enough? comparing data from twitters streaming api with twitters firehose. Proceedings of ICWSM.

Mustafaraj, E., Finn, S., Whitlock, C., and Metaxas, P. T. (2011). Vocal minority versus silent majority: Discovering the opinions of the long tail. In Proc. of 3rd IEEE SocialCom, pages 103-110. IEEE.

Pew Research Center for the People and the Press (2012). Partisan polarization surges in bush, obama years. http://bit.ly/1d2HZK6.

Prior, M. (2013). Media and political polarization. Annual Review of Political Science, 16:101-127.

Sarwar, B., Karypis, G., Konstan, J., and Riedl, J. (2001). Item-based collaborative filtering recommendation algorithms. In Proc. of the 10th $W W W$, pages 285-295. ACM.

Small, H. (1973). Co-citation in the scientific literature: A new measure of the relationship between two documents. Journal of the American Society for Information Science, 24:265-269.

Suh, B., Hong, L., Pirolli, P., and Chi, E. H. (2010). Want to be retweeted? large scale analytics on factors impacting retweet in twitter network. In 2010 2nd IEEE SocialCom, pages 177-184. IEEE.

von Ahn, L., Liu, R., and Blum, M. (2006). Peekaboom: a game for locating objects in images. In Proc. of the SIGCHI Conf. on Human Factors in Computing Systems, CHI '06, pages 55-64, New York, NY, USA. ACM.

Wellesley Trails Group (2014). Retweets indicate agreement, endorsement, trust: A meta-analysis of published twitter research. Forthcoming. 